\title{
Letter on "Sigh maneuver to enhance assessment of fluid responsiveness during pressure support ventilation"
}

\author{
Khaoula Meddeb ${ }^{1,2}$ and Mohamed Boussarsar ${ }^{1,2^{*}}$ (D) \\ This comment refers to the article available at https://doi.org/10.1186/s13054-018-2294-4
}

For the past years, sigh maneuver was mainly studied as one of many techniques to lung recruitment in acute respiratory distress syndrome [1]. Hemodynamic effects observed during these techniques were mainly related to the increase of intrathoracic pressures [2].

We read, with great attentiveness, the recent article by Messina et al. [3], a prospective bi-centric interventional study on sigh maneuver as a tool to assess fluid responsiveness during pressure support ventilation (PSV). The study was conducted among 40 critically ill patients with a stable ventilatory PSV pattern and requiring volume expansion. Variations in systolic arterial pressure (SAP), pulse pressure (PP), and stroke volume index (SVI) were assessed consequent to random application of 4-s sighs at three different inspiratory pressures; $15 \mathrm{cmH}_{2} \mathrm{O}, 25 \mathrm{cmH}_{2} \mathrm{O}$, and $35 \mathrm{cmH}_{2} \mathrm{O}$. The authors concluded that the analysis of the slope for SAP after the application of three successive sighs and the nadir of PP after Sigh35, reliably predict fluid responsiveness [3].

Perel et al. published a previous study suggesting that a standardized ventilatory maneuver consisting of a series of successive incremental pressure-controlled breaths may be useful in guiding fluid therapy in ventilated patients [4]. Certainly, Messina et al. displayed a meticulous approach to applying the presented method concluding to compelling results; however, a few questions could be raised.
First, the authors' choice to use incremental increase in pressure support as a way to implement the sigh maneuver may be reasonable for patients under pressure support ventilation; nonetheless, this method has some limits. Setting pressure requires flow and volume monitoring which can be challenging. In fact, depending on underlying respiratory mechanics, flow varies greatly to maintain identical high pressure leading to volume fluctuation. For instance, in patients with resistive respiratory mechanics, setting pressure at $35 \mathrm{cmH}_{2} \mathrm{O}$, could possibly fail to reach a significant variation in volume capable of inducing right ventricle preload and afterload changes. It could be informative if the author shared the volume ranges generated by the different set pressures, especially at $35 \mathrm{cmH}_{2} \mathrm{O}$, and what respiratory mechanics did the study population present at the time.

Second, the study population seemed to be overly selected, patients were under low pressure support levels with no hypoxemia which leads to doubts regarding this method generalizability, especially for patients with impaired respiratory mechanics.

Although the results displayed by Messina et al. [3] seem promising, further investigations are needed especially on less selected study population.

\footnotetext{
* Correspondence: hamadi.boussarsar@gmail.com;

mohamed.boussarsar@rns.tn

${ }^{1}$ Medical Intensive Care Unit, Farhat Hached University Hospital, 4000 Sousse, Tunisia

${ }^{2}$ Research Laboratory No. LR12SP09, Heart Failure, Faculty of Medicine of

Sousse, University of Sousse, Sousse 4000, Tunisia
}

(c) The Author(s). 2019 Open Access This article is distributed under the terms of the Creative Commons Attribution 4.0 International License (http://creativecommons.org/licenses/by/4.0/), which permits unrestricted use, distribution, and reproduction in any medium, provided you give appropriate credit to the original author(s) and the source, provide a link to the Creative Commons license, and indicate if changes were made. The Creative Commons Public Domain Dedication waiver (http://creativecommons.org/publicdomain/zero/1.0/) applies to the data made available in this article, unless otherwise stated. 


\title{
Authors' response
}

\author{
Antonio Messina, Davide Colombo, Maurizio Cecconi and Paolo Navalesi
}

Thank you for giving us the chance to reply to the valuable comments raised by Meddeb regarding our article [3]. The number of ventilated intensive care unit (ICU) patients, with spontaneous breathing activity, is overall increasing [5]. In this subgroup of patients, the dynamic preload indices are often not applicable.

To the best of our knowledge, this is the first hemodynamic test specifically designed for ICU patients undergoing pressure support ventilation (PSV). As pointed out by Meddeb and Boussarsar, the slope analysis of systolic pressure changes after the delivery of consecutive and incremental pressure-controlled inspiratory breaths has been previously tested in postsurgical patients without spontaneous breathing activity $[4,6]$. Our results show that this approach could be useful also during PSV, being however, far from bedside application.

The increase in intrathoracic pressure occurring during the sigh increases lung volume to an extent that varies depending on the extra pressure applied to the airway and on the mechanical properties of the respiratory system. The higher the increase in Paw and the lower the respiratory system impedance, the larger the volume generated during the sigh. We did not measure these changes, assuming that, in a homogenous population, irrespective to the absolute volume obtained by the sigh, the final hemodynamic effect would be comparable. For sure, in some patients the application of standard sigh maneuver of $35 \mathrm{cmH}_{2} 0$ for $4 \mathrm{~s}$ could trigger a drop of pulse pressure and stroke volume insufficient (leading to false negative results) or excessive (leading to false positive results) to assess fluid responsiveness. In this "proof of concept" study, we set the highest level of inspiratory support, according to the harmless maneuver already used by Patroniti et al. in ICU patients with early acute respiratory distress syndrome [7]. Moreover, as pointed out in their Letter and as stated in our paper, some inclusion and exclusion criteria of our study would limit the external validity of the results. This choice was made to reduce the risk of bias due to the number of potential interferences between a beat-to-beat analysis and the variable respiratory pattern of ICU patients undergoing partial ventilatory support. We hope that our results would encourage further investigations in this group of patients.

\section{Abbreviations}

PP: Pulse pressure; PSV: Pressure support ventilation; SAP: Systolic arterial pressure; SVI: Stroke volume index

\section{Funding}

No one

Availability of data and materials

NA

Authors' contributions

Both authors contributed equally to the following: (1) substantial contributions to conception and design, analysis, and interpretation of data; (2) drafting the article or revising it critically for intellectual content, and (3)

final approval of the version to be published.

Ethics approval and consent to participate

NA

Consent for publication

NA

\section{Competing interests}

The authors declare that they have no competing interests.

\section{Publisher's Note}

Springer Nature remains neutral with regard to jurisdictional claims in published maps and institutional affiliations.

Received: 7 March 2019 Accepted: 25 April 2019

Published online: 24 May 2019

References

1. Pelosi $P$, Cadringher $P$, Bottino N, Panigada M, Carrieri F, Riva E, et al. Sigh in acute respiratory distress syndrome. Am J Respir Crit Care Med. 1999;159(3): 872-80

2. Biais M, Lanchon R, Sesay M, Le Gall L, Pereira B, Futier E, et al. Changes in stroke volume induced by lung recruitment maneuver predict fluid responsiveness in mechanically ventilated patients in the operating room. Anesthesiology. 2017;126(2):260-7.

3. Messina A, Colombo D, Barra F, Cammarota G, De Mattei G, Longhini F, et al. Sigh maneuver to enhance assessment of fluid responsiveness during pressure support ventilation. Crit Care. 2019;23(31)

4. Perel A, Minkovich L, Preisman S, Abiad M, Segal E, Coriat P. Assessing fluidresponsiveness by a standardized ventilatory maneuver: the respiratory systolic variation test. Anesth Analg. 2005:100(4):942-5.

5. Esteban A, Frutos-Vivar F, Muriel A, Ferguson ND, Penuelas O, Abraira V, et al. Evolution of mortality over time in patients receiving mechanical ventilation. Am J Respir Crit Care Med. 2013;188:220-30.

6. Preisman S, Kogan S, Berkenstadt H, Perel A. Predicting fluid responsiveness in patients undergoing cardiac surgery: functional haemodynamic parameters including the respiratory systolic variation test and static preload indicators. Br J Anaesth. 2005;95:746-55.

7. Patroniti N, Foti G, Cortinovis B, Maggioni E, Bigatello LM, Cereda M, et al. Sigh improves gas exchange and lung volume in patients with acute respiratory distress syndrome undergoing pressure support ventilation. Anesthesiology. 2002;96:788-94. 